\title{
Mean Arterial Blood Pressure Correlates with Neurological Recovery after Human Spinal Cord Injury: Analysis of High Frequency Physiologic Data
}

\author{
Gregory Hawryluk, 1,3,4 William Whetstone, ${ }^{2}$ Rajiv Saigal,,4 Adam Ferguson,, \\ Jason Talbott, ${ }^{5}$ Jacqueline Bresnahan,, ${ }^{3,4}$ Sanjay Dhall, ${ }^{3,4}$ Jonathan Pan, ${ }^{6}$ \\ Michael Beattie, ${ }^{3,4}$ and Geoffrey Manley ${ }^{3,4}$
}

\begin{abstract}
Current guidelines for the care of patients with acute spinal cord injuries (SCIs) recommend maintaining mean arterial pressure (MAP) values of $85-90 \mathrm{~mm} \mathrm{Hg}$ for 7 days after an acute SCI however, little evidence supports this recommendation. We sought to better inform the relationship between MAP values and neurological recovery. A computer system automatically collected and stored q1 min physiological data from intensive care unit monitors on patients with SCI over a 6-year period. Data for 100 patients with acute SCI were collected. 74 of these patients had American Spinal Injury Association Impairment Scale (AIS) grades determined by physical examination on admission and at time of hospital discharge. Average MAP values as well as the proportion of MAP values below thresholds were explored for values from $120 \mathrm{~mm} \mathrm{Hg}$ to $40 \mathrm{~mm} \mathrm{Hg}$ in $1 \mathrm{~mm} \mathrm{Hg}$ increments; the relationship between these measures and outcome was explored at various time points up to 30 days from the time of injury. A total of $994,875 \mathrm{q} 1 \mathrm{~min}$ arterial line blood pressure measurements were recorded for the included patients amid 1,688,194 min of recorded intensive care observations. A large proportion of measures were below $85 \mathrm{~mm} \mathrm{Hg}$ despite generally acceptable average MAP values. Higher average MAP values correlated with improved recovery in the first 2-3 days after SCI while the proportion of MAP values below the accepted threshold of $85 \mathrm{~mm} \mathrm{Hg}$ seemed a stronger correlate, decreasing in strength over the first 5-7 days after injury. This study provides strong evidence supporting a correlation between MAP values and neurological recovery. It does not, however, provide evidence of a causal relationship. Duration of hypotension may be more important than average MAP. It provides support for the notion of MAP thresholds in SCI recovery, and the highest MAP values correlated with the greatest degree of neurological recovery. The results are concordant with current guidelines in suggesting that MAP thresholds $>85 \mathrm{~mm} \mathrm{Hg}$ may be appropriate after acute SCI.
\end{abstract}

Key words: blood pressure; mean arterial pressure; neurocritical care; neuroprotection; outcome; recovery; secondary injury; spinal cord injury

\section{Introduction}

$\mathbf{S}^{\mathrm{n}}$ PINAL CORD INJURY (SCI) leaves patients with often profound deficits of motor, sensory, sexual, and sphincter function. In recent decades, we have learned much about molecular secondary injury processes that cause progressive, delayed damage to the injured spinal cord ${ }^{1-3}$; however, we remain without a safe and efficacious therapeutic agent that targets them. ${ }^{4,5}$ We have also learned much of secondary insults such as hypoxia and hypotension that occur at the level of the organism and serve to exacerbate the injury to the spinal cord. ${ }^{6-9}$ Attention to preventing or aggressively treating secondary insults is currently the mainstay of care that follows SCI. ${ }^{10-13}$
In 2002, the first guidelines for the management of acute SCI were published. ${ }^{12}$ These guidelines recommended at the option level that hypotension-defined as a systolic blood pressure $<90 \mathrm{~mm} \mathrm{Hg}$ - should be avoided and that a mean arterial blood pressure (MAP) of $85-90 \mathrm{~mm} \mathrm{Hg}$ should be targeted in the first 7 days after SCI. These recommendations were essentially unchanged in the 2013 update of the guidelines. ${ }^{13}$ A small, heterogenous group of uncontrolled, underpowered studies supports this recommendation ${ }^{14}$; to date, no study provides better than class III evidence supporting blood pressure augmentation for acute SCI. In all relevant publications to date, blood pressure augmentation was merely part of an aggressive management protocol confounding the relationship between MAP and outcome. ${ }^{15-19}$ Moreover,

\footnotetext{
${ }^{1}$ Department of Neurosurgery, University of Utah, Salt Lake City, Utah.

Departments of ${ }^{2}$ Emergency Medicine, ${ }^{3}$ Brain and Spinal Cord Injury Center (BASIC), ${ }^{4}$ Neurosurgery, ${ }^{5}$ Radiology, and ${ }^{6}$ Anaesthesia, University of California, San Francisco, San Francisco, California.
} 
comparisons have been made to historical controls in all cases. The current recommendations are largely based on the findings of a pilot study conducted by Vale and associates. ${ }^{17}$ The result is that hospitals devote substantial resources to monitoring and augmenting blood pressure in patients who may otherwise be suitable for treatment in less resource-intensive environments, despite only modest evidence supporting this practice.

Advances in computing now allow the continuous collection and storage of high frequency physiological data and the opportunity to study the impact of this physiology with substantially greater precision than was possible previously. Our group developed a system that collected and stored data once per minute for all patients monitored in the intensive care unit (ICU). This system automatically collected data for every patient with SCI admitted to San Francisco General Hospital (SFGH) over a 6-year period affording the opportunity to study the relationship between high-frequency physiological data and outcome. Although this correlative approach does not establish causation, it provides significant insights into this relationship.

\section{Methods}

\section{Patient demographics and management}

SFGH is the only Level 1 trauma center in San Francisco and the northern San Francisco peninsula. The hospital provides care for a high volume of patients with neurotrauma and polytrauma. Patients with SCIs were admitted to the neurosurgical ICU, and a MAP of at least $85 \mathrm{mmHg}$ was targeted for 5 days after the injury, similar to published guidelines $^{12}$ and the protocol of Wolf and colleagues. ${ }^{16}$ Pharmacological agents were administered if needed to achieve this goal. ${ }^{20}$ In $26.8 \%$ of patients, the first pressor started was phenylephrine. In $48.5 \%$, the first pressor used was dopamine. In $3.1 \%$, dopamine and phenylephrine were started concurrently. In $1.0 \%$, levophed was the first used vasopressor. In $23 \%$ of patients, a second vasopressor was needed to meet the MAP targets. A detailed study of vasopressor use in an overlapping subset of SCI patients from San Francisco General Hospital has been published recently. ${ }^{20}$

Patients were identified retrospectively for this analysis. Demographic data were collected from the patients' health records as shown in Table 1. American Spinal Injury Association Impairment Scale (AIS) grades were computed based on detailed neurological examinations performed on presentation but after resuscitation, and just before discharge. ${ }^{21}$ The AIS grade improvement was calculated. Because of the limited neurological recovery known to occur in patients with AIS grade A injuries post-resuscitation, analyses were planned with and without these patients included $a$ priori.

\section{Data collection}

Our institution developed a research ethics board approved computerized data acquisition system in conjunction with Aristein Bioinformatics LLC, which collects and stores data from the patients' bedside monitor in the neurosurgical ICU. The same system was used to collect physiological data in nonhuman experimentation as described previously by our group. ${ }^{22}$ Variables displayed on the

Table 1. Characteristics of Studied Patients based on Degree of Neurological Improvement

\begin{tabular}{|c|c|c|c|c|c|}
\hline & $\begin{array}{l}\text { No outcome } \\
\text { data }(\mathrm{n}=26)\end{array}$ & $\begin{array}{l}\text { No improvement } \\
(\mathrm{n}=35)\end{array}$ & $\begin{array}{l}1 \text { AIS point improvement } \\
(\mathrm{n}=23)\end{array}$ & $\begin{array}{c}>1 \text { AIS point } \\
\text { improvement }(\mathrm{n}=13)\end{array}$ & $\mathrm{p}$ value \\
\hline & $43.0 \pm 16.1$ & $42.5 \pm 19.0$ & $50.2 \pm 22.3$ & $52.2 \pm 18.8$ & 0.434 \\
\hline Sex & $20 \mathrm{M}, 6 \mathrm{~F}$ & $28 \mathrm{M}, 7 \mathrm{~F}$ & $15 \mathrm{M}, 8 \mathrm{~F}$ & $10 \mathrm{M}, 3 \mathrm{~F}$ & 0.639 \\
\hline ISS & $27.6 \pm 16.4$ & $30.1 \pm 14.7$ & $25.4 \pm 14.3$ & $25.6 \pm 9.7$ & 0.622 \\
\hline AIS A & $?$ & $23(65.7 \%)$ & $3(13.0 \%)$ & $3(23.1 \%)$ & $<0.0001$ \\
\hline AIS B & $?$ & $1(2.8 \%)$ & $1(4.3 \%)$ & $3(23.1 \%)$ & 0.093 \\
\hline AIS C & $?$ & $3(8.6 \%)$ & $6(26.1 \%)$ & $4(30.8 \%)$ & 0.131 \\
\hline AIS D & $?$ & $5(14.3)$ & $11(47.8 \%)$ & $2(15.4 \%)$ & 0.016 \\
\hline AIS E & $?$ & $0(0 \%)$ & $2(8.7 \%)$ & $1(7.7 \%)$ & 0.995 \\
\hline Surgery & $4(15.4 \%)$ & $33(94.3 \%)$ & $19(82.6 \%)$ & $13(100 \%)$ & $<0.0001$ \\
\hline Timing of surgery & $24.0 \mathrm{~h} \pm 32.5$ & $36.4 \mathrm{~h} \pm 32.5$ & $42.9 \mathrm{~h} \pm 75.8$ & $42.0 \mathrm{~h} \pm 35.5$ & 0.917 \\
\hline Total hospital days & $47.3 \pm 48.1$ & $26.8 \pm 37.0$ & $17.9 \pm 13.9$ & $56.2 \pm 49.9$ & 0.074 \\
\hline Total measurements & $17421.3 \pm 27133.5$ & $15671.1 \pm 14970.9$ & $12946.4 \pm 14874.4$ & $21958.5 \pm 22254.0$ & 0.200 \\
\hline Penetrating & $0(0 \%)$ & $8(22.8 \%)$ & $2(8.7 \%)$ & $0(0 \%)$ & 0.006 \\
\hline Cervical & $1(20 \%)$ & $18(54.5 \%)$ & $16(72.7 \%)$ & $12(92.3 \%)$ & 0.080 \\
\hline Thoracic & $0(0 \%)$ & $13(39.3 \%)$ & $3(13.6 \%)$ & $1(7.7 \%)$ & 0.171 \\
\hline Lumbar & $4(80 \%)$ & $1(3.0 \%)$ & $2(9.1 \%)$ & $0(0 \%)$ & 0.024 \\
\hline Required two vasopressors & $1(20 \%)$ & $11(31.4 \%)$ & $5(21.7 \%)$ & $3(23.1 \%)$ & 0.927 \\
\hline
\end{tabular}

AIS, ASIA Impairment Scale ${ }^{1-3}$; here, AIS A-E denote the post-resuscitation score; ISS $=$ Injury Severity Score. ${ }^{4}$

Characteristics of analyzed patients are shown with grouping based on change in neurological function by time of discharge. For continuous data means are presented \pm standard deviation. For categorical data, frequencies are presented as well as percentage of patients for whom data were available. Three patients who exhibited neurological worsening were excluded because of small sample size $(n=3)$. The $p$ values reflect the results of univariate statistical analysis. Analysis of variance was performed for continuous data, and binomial logistic regression was used for categorical variables. Statistically significant values are italicized.

1. Marino, R.J., Barros, T., Biering-Sorensen, F., Burns, S.P., Donovan, W.H., Graves, D.E., Haak, M., Hudson, L.M., and Priebe, M.M. (2003). International standards for neurological classification of spinal cord injury. J. Spinal Cord Med. 26, Suppl 1, S50-S56.

2. Kirshblum, S.C., Burns, S.P., Biering-Sorensen, F., Donovan, W., Graves, D.E., Jha, A., Johansen, M., Jones, L., Krassioukov, A., Mulcahey, M.J., Schmidt-Read, M., and Waring, W. (2011). International standards for neurological classification of spinal cord injury (revised 2011). J. Spinal Cord Med. 34, 535-546.

3. Waring, W.P., 3rd, Biering-Sorensen, F., Burns, S., Donovan, W., Graves, D., Jha, A., Jones, L., Kirshblum, S., Marino, R., Mulcahey, M.J., Reeves, R., Scelza, W.M., Schmidt-Read, M., and Stein, A. (2010). 2009 review and revisions of the international standards for the neurological classification of spinal cord injury. J. Spinal Cord Med. 33, 346-352.

4. Baker, S.P., O’Neill, B., Haddon, W., Jr., and Long, W.B. (1974). The injury severity score: a method for describing patients with multiple injuries and evaluating emergency care. J. Trauma 14, 187-196. 
bedside monitor were collected at 1 minute intervals, and for this work, MAP values as measured with an arterial line were analyzed. The duration of arterial line blood pressure monitoring was based solely on medical necessity as judged by the treating intensivist.

Data were recorded continuously and automatically from every bedside monitor in the neurosurgical ICU between 2005 and 2011 and stored on a server in a fashion adherent to patient privacy regulations. Data acquisition with this system initiates automatically as soon as patient data appear on the bedside monitor. The time of the first recorded observation in the collection system was denoted as time " 1 ". Note that this is distinct from the time of injury or the first arterial line MAP measurement.

\section{Data analysis}

MAP values were analyzed with the assistance of Matlab. We designed a program to extract MAP values for each patient stored in individual Excel files. It calculated average MAP values between periods specified by the analyst. It was also programmed to count the number of epochs with MAP values below specified thresholds between specified periods. We selected to analyze blood pressures below 80 different thresholds from 120 to $40 \mathrm{~mm} \mathrm{Hg}(1 \mathrm{mmHg}$ increments). The Matlab program was checked for errors by comparing results with those generated using Microsoft Excel. The proportion of values below thresholds was analyzed to account for different numbers of observations between patients. Microsoft Excel and PowerPoint were used to graph the data and Photoshop CS2 was used to combine images for figures. Error bars represent standard error of the mean in all cases.

\section{Statistical analysis}

SPSS v21 software was used for statistical analyses. Analysis of variance was used as a first step in the analysis of average values from continuous data; if a difference between groups was demonstrated, the Tukey and Bonferroni post hoc tests were performed that adjust for multiple comparisons. Binomial logistic regression was used to analyze dichotomous categorical data. For average values of continuous data, the " $n$ " was considered to be the total number of observations. Where proportions were analyzed, a single proportion was calculated for each patient, and the " $n$ " was thus the number of patients in each group.

\section{Results}

\section{Characteristics of included patients}

One hundred patients with SCI were identified with continuous physiological data recordings. These patients had a total of $1,688,194$ minutes of recorded observation corresponding to 1172.4 days of total observation. A total of $994,875 \mathrm{q} 1 \mathrm{~min}$ MAP measurements were recorded corresponding to 690.1 days of MAP observations. We restricted our analysis to those values recorded in the first 30 days of hospitalization.

Of the 100 patients, it was possible to calculate the change in AIS grade between post-resuscitation and pre-discharge values for 74. Of these, three patients experienced neurological worsening, 35 exhibited no change in AIS grade, 23 had improved one AIS grade, and 13 improved more than one AIS grade. There were 27 patients who had AIS grade A injuries; when removed from the dataset, 2 patients experienced neurological worsening, 12 exhibited no change in AIS grade, 21 improved one AIS grade, and 10 exhibited more than 1 grade of improvement. The neurological improvement seen in our study was comparable with other recent publications. ${ }^{23,24}$ We excluded patients with neurological worsening from subgroup analyses given that robust conclusions could not be generated and the fact that the two patients who remained
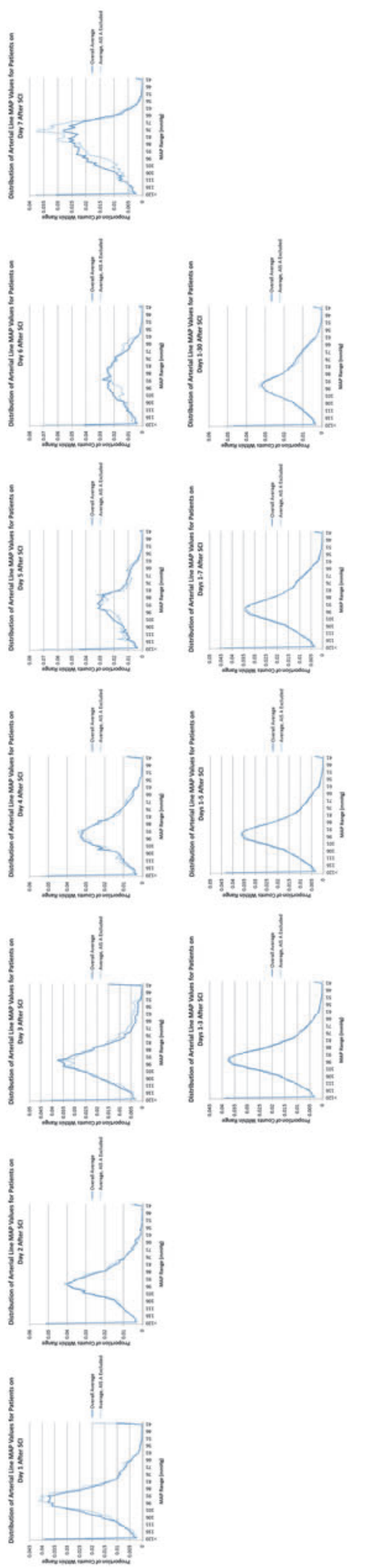
could not be included in statistical analyses when AIS grade A patients were excluded.

\section{Patient demographics}

Characteristics of studied patients are recorded in Table 1. Patients with penetrating SCI were significantly less likely to improve neurologically ( $p=0.006$, binomial logistic regression). Patients with lumbar injuries were significantly less likely to have outcome data collected for analysis ( $p=0.024$, binomial logistic regression). Although not significant, patients achieving $>1$ AIS grade improvement had substantially longer periods of observation in the ICU and a longer period of hospitalization potentially providing greater opportunity for neurological improvement than in other groups. Although the degree of neurological improvement is
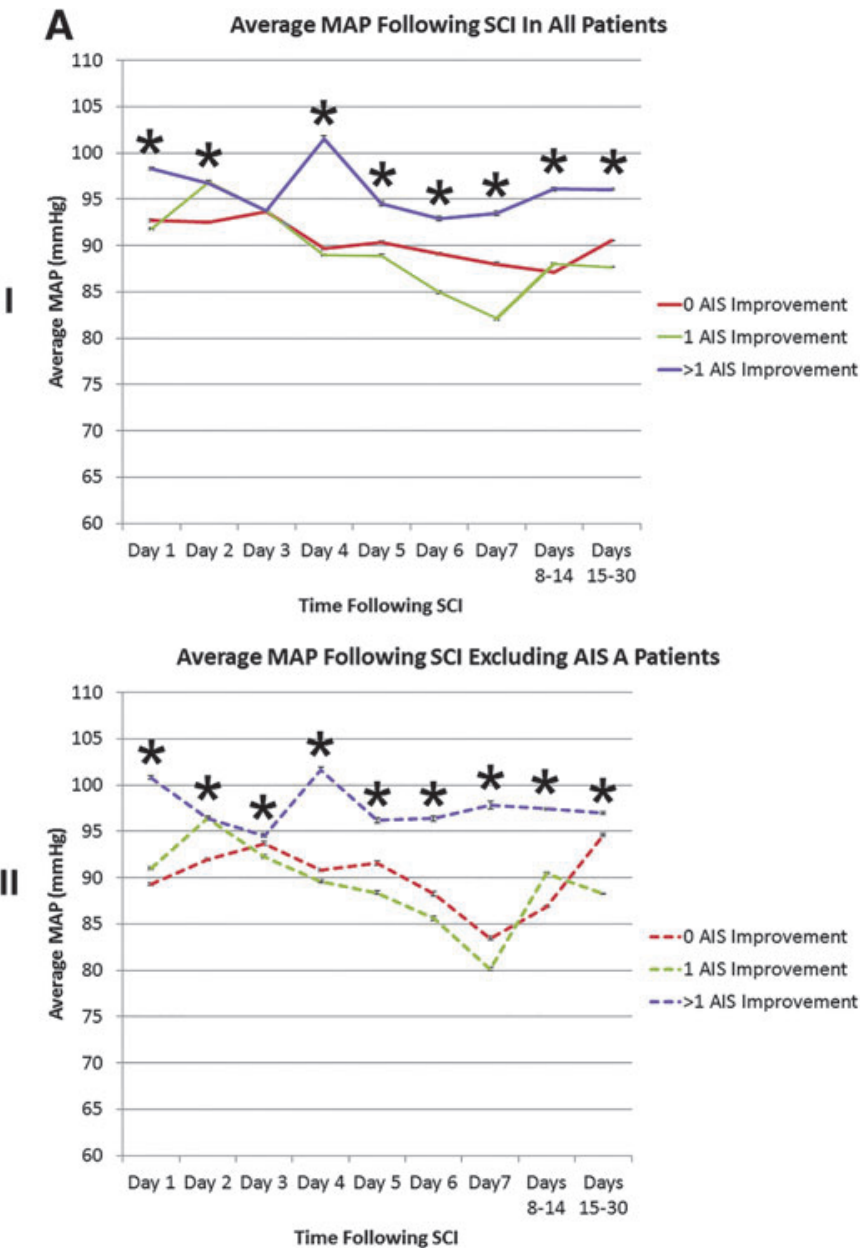

positively correlated with length of stay, the $\mathrm{R}^{2}$ value is only 0.0484 , indicating that the strength of this confound is quite weak (Supplementary Fig. 1; see online supplementary material at ftp.liebertpub.com).

Of note, $100 \%$ of patients with outcome data available needed pressor administration to achieve MAP goals. An approximately equal number of patients in each group needed a second vasopressor for blood pressure augmentation. Significantly fewer patients with missing outcome data were documented as having undergone surgery. Of those patients initially AIS grade A, a significantly greater proportion of patients exhibited no neurological improvement than some improvement. Of those patients initially AIS grade D, a significantly greater proportion of patients exhibited a single grade of neurological improvement than no improvement.

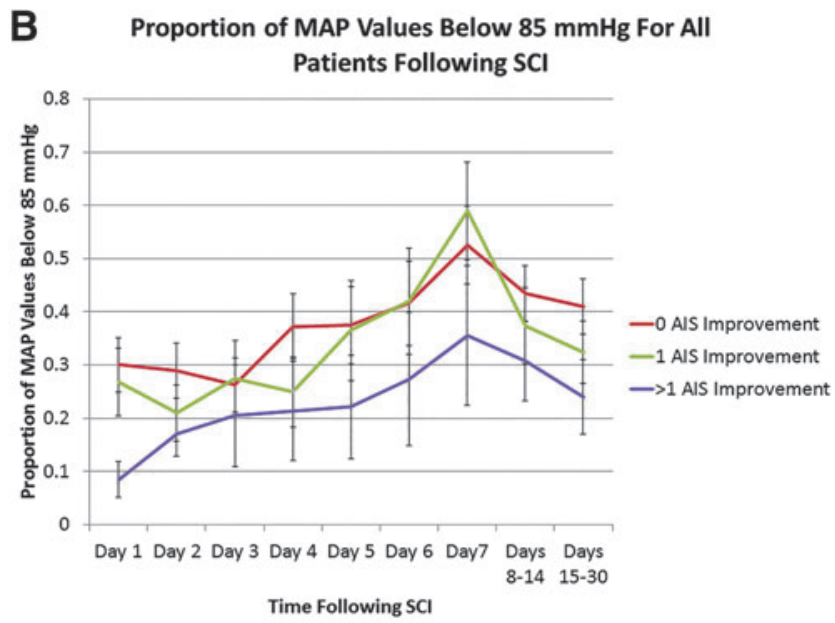

Proportion of MAP Values Below $85 \mathrm{mmHg}$ Excluding AIS A Patients Following $\mathrm{SCl}$

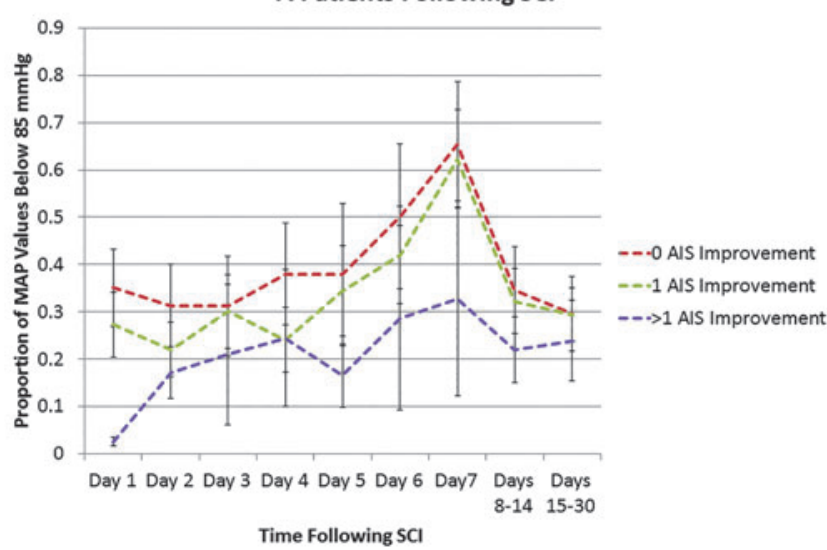

FIG. 2. Average mean arterial pressure (MAP) and proportion of values $<85 \mathrm{~mm} \mathrm{Hg}$ are associated with outcome early after spinal cord injury in a noncumulative analysis. In (A), average MAP values are plotted in relation to time subsequent to intensive care unit admission. Values were measured with an arterial line. In (B), the proportion of MAP values below the lower limit of the recommended blood pressure range $(85 \mathrm{~mm} \mathrm{Hg})$ are plotted. In (I), all patients with outcome data are plotted, while in (II), patients known to be American Spinal Injury Association Impairment Scale (AIS) grade A at final neurological examination are excluded. The latter case is denoted with dashed lines. For (A), the " $n$ " used in statistical testing was the number of blood pressure measures, while in (B), it was the number of patients.

SCI, spinal cord injury.

*Denotes significance on analysis of variance performed at each time point. Error bars represent standard error.

I: For the group with 0 AIS grade improvement, $n=35$ patients; the group with 1 AIS grade improvement, $n=23$ patients, and the group with $>1$ AIS grade improvement, $n=13$ patients.

II: For the group with 0 AIS grade improvement, $n=13$ patients; the group with 1 AIS grade improvement, $n=21$ patients. and the group with $>1$ AIS grade improvement, $n=10$ patients. Color image is available online at www.liebertpub.com/neu 
A Average MAP Values for Spinal Cord Injury Patients in the First Three Days Following Injury

I

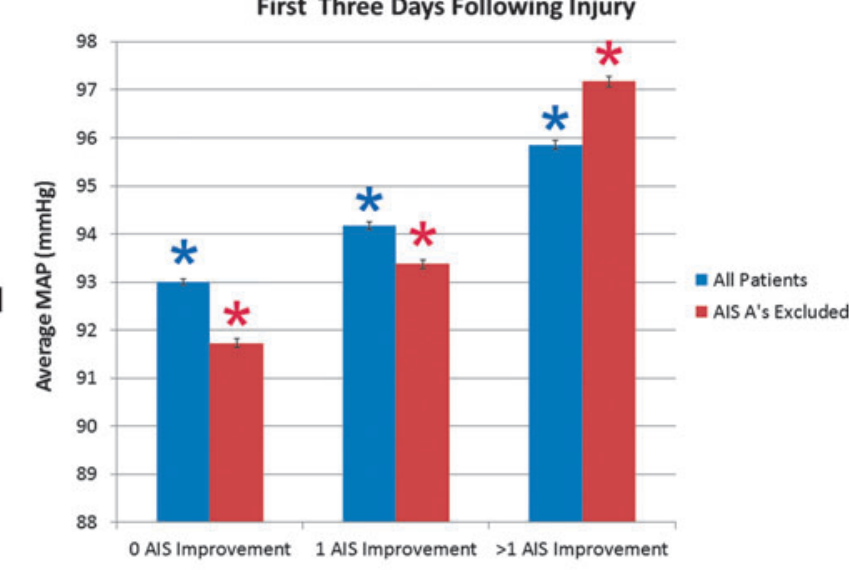

Average MAP Values for Spinal Cord Injury Patients in the First Seven Days Following Injury

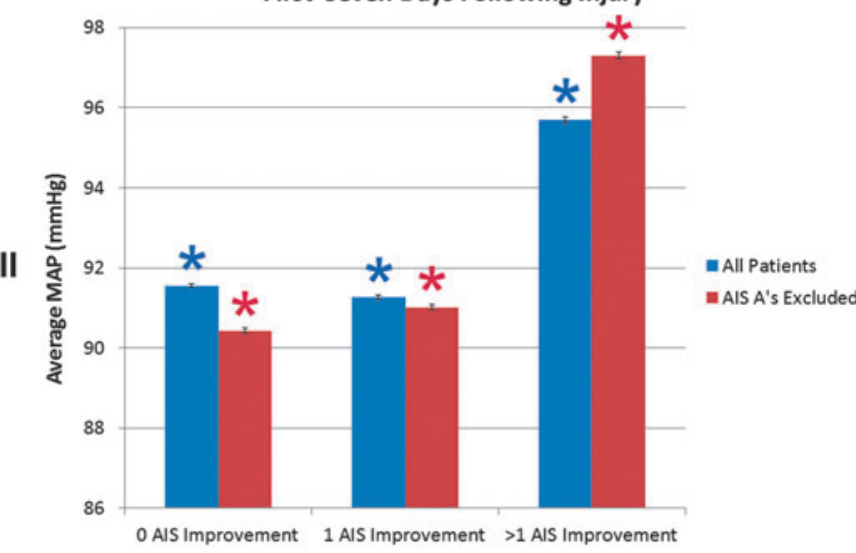

Average MAP Values for Spinal Cord Injury Patients in the First Thirty Days Following Injury

III

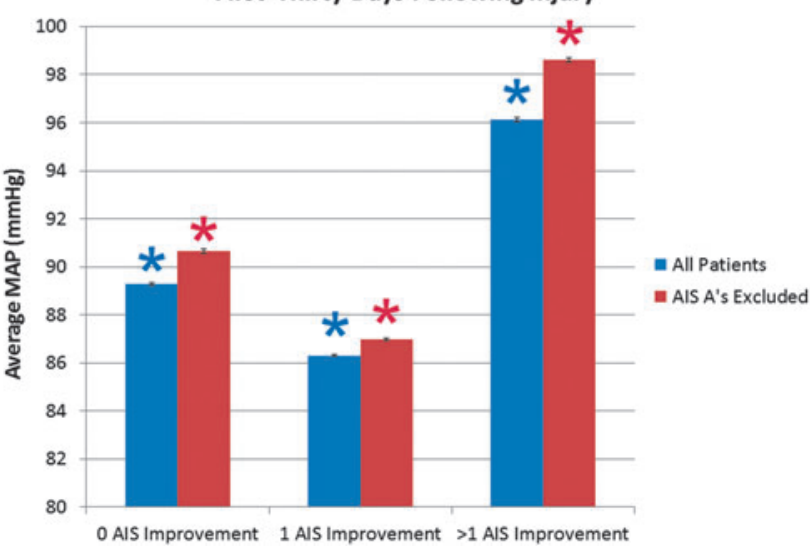

B

Proportion of MAP Values Below $85 \mathrm{mmHg}$ for Spinal

Cord Injury Patients in the First Three Days Following Injury

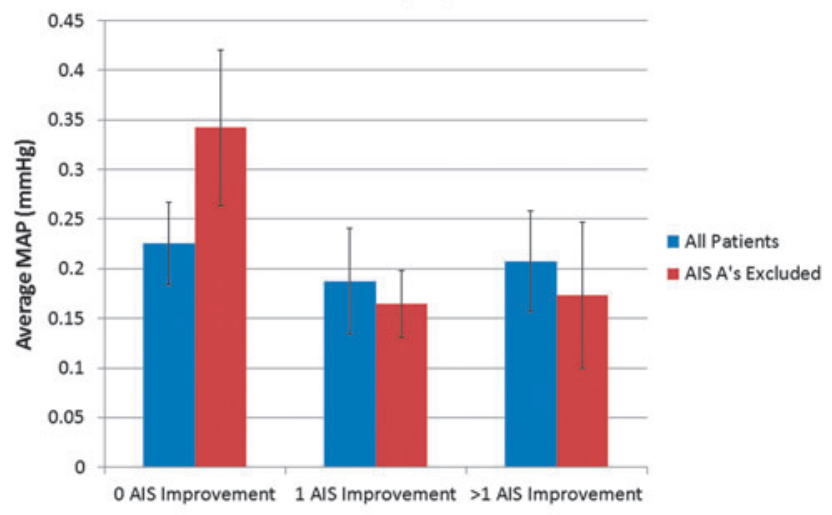

Proportion of MAP Values Below $85 \mathrm{mmHg}$ for Spinal Cord Injury Patients in the First Seven Days Following Injury

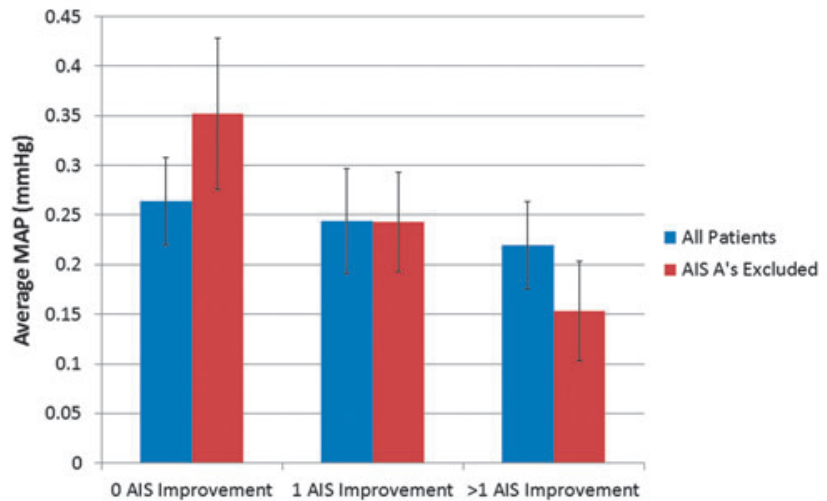

Proportion of MAP Values Below $85 \mathrm{mmHg}$ for Spinal Cord Injury Patients in the First Thirty Days Following Injury

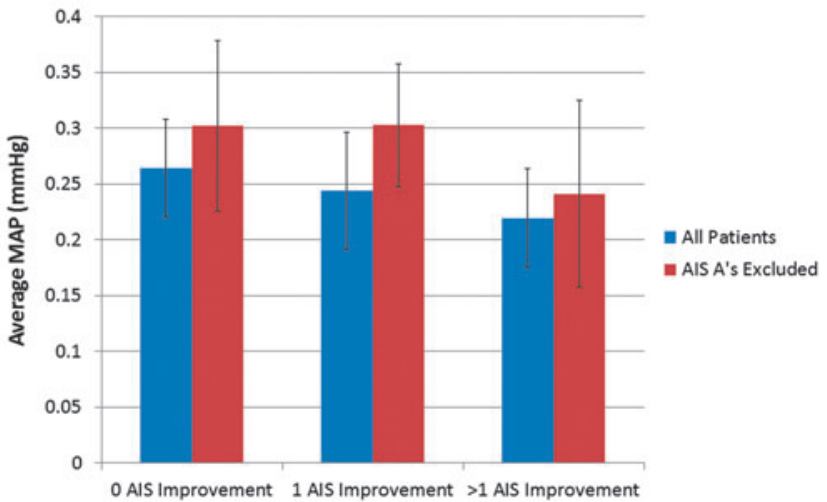

FIG. 3. Average mean arterial pressure (MAP) and proportion of values $<85 \mathrm{~mm} \mathrm{Hg}$ are significantly associated with outcome early after spinal cord injury in a cumulative analysis. In (A), average MAP values are plotted. In (B), the proportion of MAP values below the lower limit of the recommended blood pressure range $(85 \mathrm{~mm} \mathrm{Hg})$ are plotted. Values were obtained from arterial line measures, and times reflect the interval since intensive care unit admission. In (I), values for days 1-3 post-spinal cord injury are presented. In (II), values for days 1-7 post-spinal cord injury are presented. In (III), values for days 1-30 post spinal cord injury are presented. For data including all patients (blue), the group with 0 AIS grade improvement, $n=35$ patients; the group with 1 AIS grade improvement, $n=23$ patients; and the group with $>1$ AIS grade improvement, $n=13$ patients. For data excluding patients AIS grade A on their final neurological examination (red), the group with 0 AIS grade improvement, $n=13$ patients; the group with 1 AIS grade improvement, $n=21$ patients, and the group with $>1$ AIS grade improvement, $n=10$ patients.

*Denotes a statistical difference from all other groups on post-hoc Bonferroni testing subsequent to a significant overall analysis of variance. Error bars represent standard error. For $(\mathbf{A})$, the " $n$ " used in statistical testing was the number of blood pressure measures, while in (B), it was the number of patients. Color image is available online at www.liebertpub.com/neu 


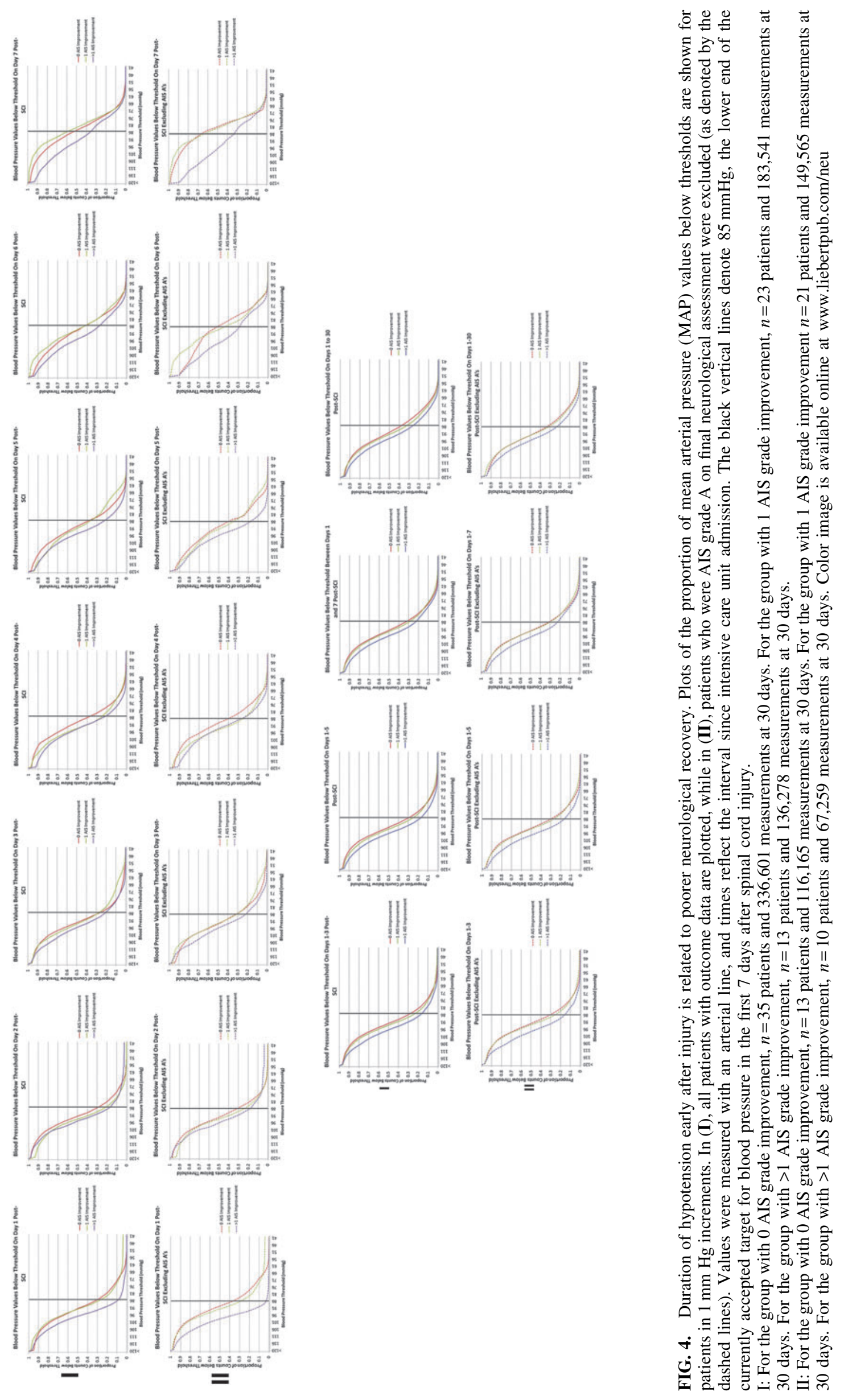




\section{Distribution of MAP for all patients after $S C I$}

The distribution of MAP values for all 100 patients at various time points subsequent to SCI is demonstrated in Figure 1. Figure 1 presents a frequency plot of the proportion of counts in $1 \mathrm{~mm} \mathrm{Hg}$ blood pressure ranges between 40 and $120 \mathrm{~mm} \mathrm{Hg}$. Plots are also shown with the exclusion of patients who were AIS grade A on final neurological examination (dashed lines). While most measured MAP values were above the $85 \mathrm{~mm} \mathrm{Hg}$ treatment threshold, $42.1 \%$ of all recorded MAP values within 30 days of injury were below $85 \mathrm{~mm} \mathrm{Hg}$. In the first 7 days, $28.8 \%$ of measures were below the recommended treatment threshold of $85 \mathrm{~mm} \mathrm{Hg}$. During the first 5 days, while an attempt was made to meet the MAP target, $24.9 \%$ of measures were below the $85 \mathrm{~mm} \mathrm{Hg}$ threshold. Exclusion of AIS grade A patients did not substantially alter the distribution of measures. Of note, the curves generally conform to a bell-shape with minimal skew; values above the mean were generally as probable as those below without apparent influence of the $85 \mathrm{~mm} \mathrm{Hg}$ threshold.

\section{Relationship between average MAP, time below MAP threshold, time from ICU admission and neurological improvement}

In Figure 2, average MAP values and the proportion of MAP values below $85 \mathrm{~mm} \mathrm{Hg}$ are plotted for groups segregated by amount of neurological improvement for various noncumulative time points subsequent to ICU admission. Average MAP values were uniformly $>85 \mathrm{~mm} \mathrm{Hg}$ during the 5 days that MAP targeting was used. Despite this, a large proportion of MAP measurements were below this threshold at every examined time point. Average MAP values are thus insensitive to episodes of hypotension or time below treatment threshold.

The group achieving $>1$ AIS grade improvement had the highest MAP and lowest proportion of measures below $85 \mathrm{~mm} \mathrm{Hg}$ at every examined time point whether or not AIS grade A patients were included in the analysis. MAP was higher in the group achieving 1 AIS grade improvement than in the group that did not improve only for the first 24-72 $\mathrm{h}$ after ICU admission. Patients achieving 1 AIS grade improvement had a lower proportion of MAP measures below $85 \mathrm{~mm} \mathrm{Hg}$ than in the group that did not improve only for the first 5-7 days after ICU admission. This difference seemed to lessen with time, however.

A cumulative analysis of values in the first 3, 7, and 30 days is presented in Figure 3. A higher average MAP correlated with outcome in the first 3 days after injury; however, by 7 days, higher MAP values were only noted in the group achieving $>1$ AIS grade improvement. The proportion of measures $<85 \mathrm{~mm} \mathrm{Hg}$ was generally associated with outcome at all time points after injury. As in Figure 2, results were similar whether or not AIS grade A patients were included or excluded from the analysis.

\section{MAP thresholds and neurological improvement after $\mathrm{SCl}$}

To explore the notion of MAP thresholds, we plotted the proportion of values below 80 different and physiologically relevant MAP thresholds for groups based on degree of neurological improvement (Fig. 4). When lines cross, diverge, or are separated, an effect of MAP on outcome is possible. The group achieving $>1$ grade of AIS grade improvement had a reduced number of measures below all examined MAP thresholds compared with groups with less improvement-and this difference was particularly marked in the first $24 \mathrm{~h}$. The lowest MAP at which patients with no improvement were distinguished from those with 1 grade of improvement was $70-75 \mathrm{~mm} \mathrm{Hg}$, suggesting that this may be the lowest blood pressure threshold associated with neurological benefit. Moreover, these two lines tended to re-converge around $95 \mathrm{~mm}$ $\mathrm{Hg}$, suggesting that values above this level are not related to the one grade of neurological improvement discerning these groups. The gap between plotted lines for the group that did not improve and that which improved one grade decreases over time, providing additional evidence that the neuroprotective effect of MAP elevation decreases with time from injury. A threshold discriminating patients with $>1$ AIS grade improvement from other groups is not suggested.

\section{Discussion}

Spinal cord ischemia is believed to play a central role in the secondary injury processes that cause delayed and progressive injury to the spinal cord after the initial-or primary-SCI has ceased. ${ }^{1,2}$ This secondary injury can be exacerbated by secondary insults such as hypotension and hypoxia, which are, unfortunately, common in patients with SCIs who frequently exhibit neurogenic and/or hemorrhagic shock..$^{8,9,18,25,26}$ Spinal cord blood flow has been subject to detailed study in animal models of SCI, and its impairment is believed to contribute to neurological injury after SCI. ${ }^{27-29}$ Moreover, patients with SCI frequently exhibit autonomic and hemodynamic instability in the first week after injury. ${ }^{30,31}$

There is thus a strong rationale for ICU monitoring and blood pressure support early after SCI. Conceptually, we can think of blood pressure support as achieving a higher than normal pressure to augment the delivery of nutrients to injured tissue or as preventing hypotension and a deficiency of nutrient supply. ${ }^{18}$ These two notions of blood pressure support should be considered separately and are worthy of independent study.

Hospitals caring for patients with SCI spend millions of dollars each year to comply with the published guidelines despite the very weak supportive data ${ }^{9}$ and known complications that can occur as a result of vasopressor administration ${ }^{20,32}$ or the bed rest with which it is associated. ${ }^{33-35}$ Our institution estimates that the cost of ICU care is $\$ 2500-\$ 4000$ a day. It is estimated that there are 12,500 SCIs in the United States annually. ${ }^{36}$ The cost of 7 days of ICU care for these patients is thus estimated to be $\$ 218,750,000$ $\$ 350,000,000$ with the cost of each day of monitoring costing $\$ 31,250,000-\$ 50,000,000$. Indeed, in this study all patients for whom outcome data were available needed vasopressor therapy to achieve the target MAP, and this is consistent with other published reports ${ }^{17}$; the need to administer vasopressors is largely implicit with following the guidelines. Given this, the notion of blood pressure augmentation as a neuroprotective strategy after acute SCI needs further study as a high priority.

The present study is unique in that it has been able to correlate high-frequency blood pressure measures from a large cohort of patients with SCI with outcome. Although it cannot provide evidence for causation, it does provide important information about the relationship. Notably, the findings of this study were robust and generally consistent irrespective of the time frame analyzed and whether or not AIS grade A patients were included. It is critical to note that a correlation between blood pressure and severity of SCI has been previously reported and is an important potential confound. ${ }^{19,30}$ Nonetheless, this study is noteworthy for suggesting that both MAP augmentation and the avoidance of hypotension could have neuroprotective effects, and it informs the time frame over which these effects may be relevant. 


\section{Literature to date}

A small number of studies provide class III evidence for observing patients in the ICU for 7 days after SCI and maintaining a target MAP > $85 \mathrm{~mm} \mathrm{Hg.}{ }^{9,12,13}$ In 1976, Zach and associates ${ }^{15}$ published a prospective study of 117 patients with SCI that reported a high level of conversion from complete to incomplete injuries when early aggressive treatment was undertaken; however, this study did not use a control group and did not specifically study nor report on the issue of MAP augmentation. In 1977 Hachen et al. reported a series of 188 patients who were rapidly admitted to intensive care and exhibited a much better mortality rate compared with statistics from $1966 .{ }^{37}$ In 1979 , Gschaedler and coworkers $^{38}$ reported a similar study involving 51 patients and similar findings.

In 1984, Tator and colleagues ${ }^{18}$ published a series of 144 patients whom they compared with historic controls. These patients were treated with aggressive efforts to avoid hypotension and hypoxia, and they received this care more rapidly than the control group. The more aggressive management protocol was associated with lower mortality, morbidity, and healthcare costs. In 1991, Wolf and associates ${ }^{16}$ studied 52 patients with bilateral cervical facet dislocations for whom they targeted a MAP $>85 \mathrm{~mm} \mathrm{Hg}$ for 5 days amid a paradigm emphasizing rapid medical and surgical treatment. The authors claimed neurological improvement; however, no comparison with controls was made. In 1993, Levi and coworkers ${ }^{19}$ instead targeted a MAP $>90 \mathrm{~mm} \mathrm{Hg}$ as part of their aggressive SCI management paradigm. In their report of 50 patients, they concluded that aggressive care reduced mortality and morbidity; however, it did not include a control group.

Vale and associates ${ }^{17}$ reported a "pilot study" of 77 patients who underwent aggressive volume expansion with Swan-Ganz catheter monitoring, and a target of MAP $>85 \mathrm{~mm} \mathrm{Hg}$ for 7 days after injury. The authors concluded that the better-than-expected outcomes seemed attributable to their emphasis on hemodynamic parameters; however, no comparison was made with a control group, and the 7-day period was picked arbitrarily. This study largely serves as the basis for the current guidelines for the acute cardiovascular management of patients with SCIs. ${ }^{12,13}$

Cohn and coworkers ${ }^{14}$ more recently reported a retrospective study of the relationship between episodes of hypotension noted in the medical record and outcomes using step-wise regression, and concluded that there may be a threshold of around $70 \mathrm{~mm} \mathrm{Hg}$ below which worse outcomes are seen. This study involved only 17 patients and had less accurate data on brief periods of hypotension, but its results are in accordance with our findings (Fig. 4).

Few strong conclusions can be drawn from the literature to date. A major limitation of these studies is a lack of evidence about patients' actual blood pressures - simply allocating a patient to a blood pressure target does not mean that the patients achieved it nor does it exclude substantial periods of hypotension. This is evidenced in our own work by the fact that nearly a third of the recorded values for our patients were below treatment threshold despite efforts to prevent this (Fig. 1, 2, 4).

Another important limitation of publications to date is that blood pressure augmentation was co-administered with other aggressive management strategies, preventing a causal relationship from being established. As well, no study made a comparison to appropriate contemporaneous controls-where a comparison was made it was to historical or previously published controls. Lastly, the distinct issues of avoiding values below threshold and elevating MAP above threshold were insufficiently distinguished by these studies.

\section{A suggestion of time frames, thresholds, and the importance of avoiding MAPs below treatment threshold}

Our study found that average MAP values correlated with outcome for only 2-3 days after ICU admission, which is noteworthy given that current guidelines recommend targeting a MAP $>85 \mathrm{~mm}$ $\mathrm{Hg}$ for 7 days after injury. The proportion of values below threshold correlated with outcome for 5-7 days after injury, although the magnitude of this relationship decreased over time (Fig. 2, 3). Indeed, the group achieving $>1$ AIS grade of improvement had a substantially lower burden of hypotension than other groups in the first $24 \mathrm{~h}$ compared with other time points (Fig. 4). Taken together, these data suggest that the duration of time below treatment threshold may have a more important influence on neurological outcome than the average MAP, ${ }^{18}$ and it provides support for the notion of blood pressure monitoring and augmentation for 5-7 days after SCI.

MAP thresholds are suggested when the plotted lines in Figure 4 diverge or cross. The gap between plotted lines for the group exhibiting no improvement and that which improved 1 AIS grade was a robust finding in our work. The divergence was generally noted around $70-75 \mathrm{~mm} \mathrm{Hg}$, and the lines tended to converge again around $90-95 \mathrm{~mm} \mathrm{Hg}$. The three groups are most robustly discriminated by a MAP around $85 \mathrm{~mm} \mathrm{Hg}$, providing support for the treatment threshold recommended in the acute SCI guidelines. ${ }^{12,13}$

Our study suggests that neurological benefit may begin around MAP values of 70-75 mm Hg, consistent with the report of Cohn and coworkers. ${ }^{14} \mathrm{~A}$ threshold associated with the achievement of $>1$ grade of AIS improvement was not clearly evident. Given the consistent suggestion of higher MAP values and less hypotension in this group, we cannot exclude the possibility that a higher MAP target may contribute to greater degrees of neurological recovery.

\section{Values below treatment threshold were frequent and yet not apparent in analysis of average MAP}

The results shown in Fig. 1-4 are remarkable in demonstrating that a large proportion of measurements in our cohort of patients were below the treatment threshold despite an attempt to maintain MAP above the treatment threshold. Moreover, these values below threshold occurred despite consistent achievement of average MAP values above the threshold. Additionally remarkable is that at most time points, the distribution of MAP values approximated a normal distribution with values below the mean as likely as those above. Fewer values below the mean would have been anticipated. This speaks to the difficulty inherent to consistently achieving a MAP $>85 \mathrm{~mm} \mathrm{Hg}$ in patients with SCI who may have neurogenic and/or hemorrhagic shock and perhaps difficulty overcoming homeostatic mechanisms.

The suggested importance of relative hypotension has important implications for trial design. If a trial randomized patients to one MAP goal or another, high frequency MAP data would be important to ensure that the targets were actually reached for a significant duration of patient care. Moreover, it would play an important role in ensuring that hypotensive episodes do not confound putative effects of the target MAPs.

\section{The clinical relevance of benefit that decreases over time}

The finding that the benefit of MAP augmentation appears to decrease over time is of great clinical significance. Vasopressor 
administration can be associated with complications such as cardiac dysrhythmia and cardiac ischemia. ${ }^{20}$ The prolonged bed rest associated with MAP targeting accentuates SCI patients' already high risk of deep venous thrombosis and may be associated with an elevated risk of pressure sores, nosocomial infections, and deconditioning. ${ }^{33-35,39-41}$ In patients who show these or other adverse effects early in their course of care, a decreased length of administration may be appropriate, given that the risks of blood pressure augmentation ${ }^{20}$ likely increase over time while the benefits seem to decrease. Moreover, these data suggest that it may be appropriate to aggressively treat patients for the first 1-2 days even if their deficits are mild or if they are presumed to be high risk for complications.

It is interesting to consider that there may also be increased benefit inherent to blood pressure augmentation at the earliest possible time after injury - in the emergency department or even by first responders, although defining the etiology of hypotension is critical before initiating pressors because mortality increases in hemorrhagic shock treated with pressors. ${ }^{42}$

\section{MAP augmentation might have a causal role in neurological recovery}

As interesting observation in this study is the fact that after cessation of MAP augmentation after the fifth day of ICU care, the group achieving a single grade of AIS improvement had a higher proportion of MAP values below threshold than the group that did not improve, while previously the converse was true (Fig. 2). This suggests that the baseline blood pressure in the group exhibiting 1 grade of improvement was inherently lower and that the vasopressor therapy in this group led to higher values early after SCI. This does not prove that MAP augmentation caused the neurological recovery, but it does suggest this could be the case. Indeed, all patients with outcome data needed vasopressor therapy to achieve their MAP targets, and approximately the same number of patients in each group needed two agents, suggesting a similar burden of baseline hypotension. Moreover, the observed relationships were consistent whether or not AIS grade A patients were included in the analyses.

Despite the limited evidence supporting MAP augmentation after SCI, future trials that could establish a causal relationship will likely need to compare the current MAP target to one that is higher because a control group involving a lower target or no target would likely be judged unethical.

\section{Limitations}

In interpreting this study, a number of caveats are important to consider. Although the physiological data were collected prospectively, the remainder of this study was performed retrospectively. We did not have sufficient long-term follow-up data to use outcomes after discharge in our analysis. We were unable to calculate an AIS grade change for nearly a quarter of the patients in our study, which reflects challenges inherent to merging multiple medical record numbers for each patient at our institution and limited availability of research personnel.

The denoted time periods begin with the onset of ICU monitoring and not time from injury; this is, however, clinically relevant. As well, we do not have a record of the time at which blood pressure augmentation was initiated nor the patients' baseline blood pressure measures. Because MAP augmentation was ceased at 5 days, we are aware of the patients' blood pressure values after cessation of therapy, which provides similar information. Accordingly, the SFGH practice of maintaining MAP goals for 5 days after SCI instead of 7 days must also be considered in the interpretation of these results.

There are a number of potential confounds in our study. A significant difference in the proportion of penetrating injuries among outcome groups is a potential confound; however, our findings were robust when patients with AIS grade A injuries at pre-discharge examination were excluded. The potential confounding effect of injury severity on MAP values has been noted. ${ }^{19,30}$ As discussed, all patients with outcome measures needed vasopressor therapy, and a similar proportion in each group needed two vasopressors to maintain MAP goals, which suggests a similar baseline burden of hypotension in each group and that differences in outcome could be related to the achieved MAP values. Also important is that the patients achieving $>1$ AIS grade of improvement stayed longer in the ICU and in the hospital on average and had greater opportunity for neurological improvement than the other groups. As discussed and illustrated in Supplemental Figure 1 (see online supplementary material at ftp.liebertpub.com), the confound associated with this effect appears to be minimal. As well, such a relationship was not evident for the group achieving 1 grade of improvement compared with the group that did not improve.

\section{Conclusions}

This is the first study to provide a detailed analysis of the relationship between high frequency MAP measures and extent of neurological improvement. Although this study cannot establish a causative relationship, it provides a wealth of information about the relationship. This study suggests that average MAP may only relate to neurological outcome in the first 2-3 days after injury. The duration of time below treatment threshold may be of greater relevance to neurological recovery-a relationship with outcome is suggested for 5-7 days after injury and the relationship seems to decrease in strength over time. These results are largely consistent with published guidelines for the management of acute SCI. Prospective study randomizing patients to different MAP targets will be an important next step for these patients.

\section{Acknowledgment}

This work was supported by DoD CDMRP grants SC090241 (W81XWH-10-1-0910) and SC120259 (W81XWH-13-1-0297) to MSB and GTM. Jonathan Pan was supported by NIH T32 GM008440.

\section{Author Disclosure Statement}

No competing financial interests exist.

\section{References}

1. Rowland, J.W., Hawryluk, G.W., Kwon, B., and Fehlings, M.G. (2008). Current status of acute spinal cord injury pathophysiology and emerging therapies: promise on the horizon. Neurosurg. Focus 25, E2.

2. Borgens, R.B., and Liu-Snyder, P. (2012). Understanding secondary injury. Q. Rev. Biol. 87, 89-127.

3. Profyris, C., Cheema, S.S., Zang, D., Azari, M.F., Boyle, K., and Petratos, S. (2004). Degenerative and regenerative mechanisms governing spinal cord injury. Neurobiol. Dis. 15, 415-436.

4. Cadotte, D.W., and Fehlings, M.G. (2011). Spinal cord injury: a systematic review of current treatment options. Clin. Orthop. Relat. Res. 469, 732-741.

5. Hawryluk, G.W., Rowland, J., Kwon, B.K., and Fehlings, M.G. (2008). Protection and repair of the injured spinal cord: a review of completed, ongoing, and planned clinical trials for acute spinal cord injury. Neurosurg. Focus 25, E14. 
6. Chu, D., Qiu, J., Grafe, M., Fabian, R., Kent, T.A., Rassin, D., Nesic, O., Werrbach-Perez, K., and Perez-Polo, R. (2002). Delayed cell death signaling in traumatized central nervous system: hypoxia. Neurochem. Res. 27, 97-106.

7. Stys, P.K. (1998). Anoxic and ischemic injury of myelinated axons in CNS white matter: from mechanistic concepts to therapeutics. J. Cereb. Blood Flow Metab. 18, 2-25.

8. Hagen, E.M., Rekand, T., Gronning, M., and Faerestrand, S. (2012). Cardiovascular complications of spinal cord injury. Tidsskr. Nor. Laegeforen. 132, 1115-1120.

9. Casha, S., and Christie, S. (2011). A systematic review of intensive cardiopulmonary management after spinal cord injury. J. Neurotrauma 28, 1479-1495.

10. Evans, L.T., Lollis, S.S., and Ball, P.A. (2013). Management of acute spinal cord injury in the neurocritical care unit. Neurosurg. Clin. N. Am. 24, 339-347.

11. Ball, P.A. (2001). Critical care of spinal cord injury. Spine (Phila Pa 1976) 26, Suppl 24, S27-S30.

12. Hadley, M.N., Walters, B.C., Grabb, P.A., Oyesiku, N.M., Przybylski, G.J., Resnick, D.K., Ryken, T.C., and Mielke, D.H. (2002). Guidelines for the management of acute cervical spine and spinal cord injuries. Clin. Neurosurg. 49, 407-498.

13. Walters, B.C., Hadley, M.N., Hurlbert, R.J., Aarabi, B., Dhall, S.S., Gelb, D.E., Harrigan, M.R., Rozelle, C.J., Ryken, T.C., and Theodore, N. (2013). Guidelines for the management of acute cervical spine and spinal cord injuries: 2013 update. Neurosurgery 60, Suppl $1,82-91$.

14. Cohen, J.A., Wright, J., McKenna, S.L., and Bushnik (2010). Impact of mean arterial blood pressure during the first seven days post spinal cord injury. Top. Spinal Cord Inj. Rehabil. 15, 96-106.

15. Zach, G.A., Seiler, W., and Dollfus, P. (1976). Treatment results of spinal cord injuries in the Swiss Parplegic Centre of Basle. Paraplegia $14,58-65$

16. Wolf, A., Levi, L., Mirvis, S., Ragheb, J., Huhn, S., Rigamonti, D., and Robinson, W.L. (1991). Operative management of bilateral facet dislocation. J. Neurosurg. 75, 883-890.

17. Vale, F.L., Burns, J., Jackson, A.B., and Hadley, M.N. (1997). Combined medical and surgical treatment after acute spinal cord injury: results of a prospective pilot study to assess the merits of aggressive medical resuscitation and blood pressure management. J. Neurosurg. 87, 239-246.

18. Tator, C.H., Rowed, D.W., Schwartz, M.L., Gertzbein, S.D., Bharatwal, N., Barkin, M., and Edmonds, V.E. (1984). Management of acute spinal cord injuries. Can. J. Surg. 27, 289-293, 296.

19. Levi, L., Wolf, A., and Belzberg, H. (1993). Hemodynamic parameters in patients with acute cervical cord trauma: description, intervention, and prediction of outcome. Neurosurgery 33, 1007-1017.

20. Inoue, T., Manley, G.T., Patel, N., and Whetstone, W.D. (2014). Medical and surgical management after spinal cord injury: vasopressor usage, early surgerys, and complications. J, Neurotrauma 31, 284-291.

21. Kirshblum, S.C., Burns, S.P., Biering-Sorensen, F., Donovan, W., Graves, D.E., Jha, A., Johansen, M., Jones, L., Krassioukov, A., Mulcahey, M.J., Schmidt-Read, M., and Waring, W. (2011). International standards for neurological classification of spinal cord injury (revised 2011). J. Spinal Cord Med. 34, 535-546.

22. Rosenthal, G., Morabito, D., Cohen, M., Roeytenberg, A., Derugin, N., Panter, S.S., Knudson, M.M., and Manley, G. (2008). Use of hemoglobin-based oxygen-carrying solution-201 to improve resuscitation parameters and prevent secondary brain injury in a swine model of traumatic brain injury and hemorrhage: laboratory investigation. J. Neurosurg. 108, 575-587.

23. Fehlings, M.G., Vaccaro, A., Wilson, J.R., Singh, A., W.Cadotte, D., Harrop, J.S., Aarabi, B., Shaffrey, C., Dvorak, M., Fisher, C., Arnold, P., Massicotte, E.M., Lewis, S., and Rampersaud, R. (2012). Early versus delayed decompression for traumatic cervical spinal cord injury: results of the Surgical Timing in Acute Spinal Cord Injury Study (STASCIS). PloS One 7, e32037.

24. Fehlings, M.G., Theodore, N., Harrop, J., Maurais, G., Kuntz, C., Shaffrey, C.I., Kwon, B.K., Chapman, J., Yee, A., Tighe, A., and McKerracher, L. (2011). A phase I/IIa clinical trial of a recombinant Rho protein antagonist in acute spinal cord injury. J. Neurotrauma 28, 787-796.

25. Yanagawa, Y., Marcillo, A., Garcia-Rojas, R., Loor, K.E., and Dietrich, W.D. (2001). Influence of posttraumatic hypoxia on behavioral recovery and histopathological outcome following moderate spinal cord injury in rats. J. Neurotrauma 18, 635-644.

26. Hall, E.D., and Wolf, D.L. (1986). A pharmacological analysis of the pathophysiological mechanisms of posttraumatic spinal cord ischemia. J. Neurosurg. 64, 951-961.

27. Sandler, A.N., and Tator, C.H. (1976). Effect of acute spinal cord compression injury on regional spinal cord blood flow in primates. J. Neurosurg. 45, 660-676.

28. Sandler, A.N., and Tator, C.H. (1976). Regional spinal cord blood flow in primates. J. Neurosurg. 45, 647-659.

29. Tator, C.H., and Fehlings, M.G. (1991). Review of the secondary injury theory of acute spinal cord trauma with emphasis on vascular mechanisms. J. Neurosurg. 75, 15-26.

30. Piepmeier, J.M., Lehmann, K.B., and Lane, J.G. (1985). Cardiovascular instability following acute cervical spinal cord trauma. Cent. Nerv. Syst. Trauma 2, 153-160.

31. Lehmann, K.G., Lane, J.G., Piepmeier, J.M., and Batsford, W.P. (1987). Cardiovascular abnormalities accompanying acute spinal cord injury in humans: incidence, time course and severity. J. Am. Coll. Cardiol. 10, 46-52.

32. Plurad, D.S., Talving, P., Lam, L., Inaba, K., Green, D., and Demetriades, D. (2011). Early vasopressor use in critical injury is associated with mortality independent from volume status. J. Trauma $71,565-572$.

33. Epstein, N.E. (2014). A review article on the benefits of early mobilization following spinal surgery and other medical/surgical procedures. Surg. Neurol. Int. 5, S66-S73.

34. Suppl 3, Biros, E., Marshall, R., Jelbart, M., Milanese, S., Gordon, S., and Galea, M.P. (2014). Prevalence and risk-factors of neurogenic heterotopic ossification in traumatic spinal cord and traumatic brain injured patients admitted to specialised units in Australia. J. Musculoskelet. Neuronal Interact. 14, 19-28.

35. Chung, W.S., Lin, C.L., Chang, S.N., Chung, H.A., Sung, F.C., and Kao, C.H. (2014). Increased risk of deep vein thrombosis and pulmonary thromboembolism in patients with spinal cord injury: a nationwide cohort prospective study. Thromb. Res. 133, 579584 .

36. The National SCI Statistical Center Center (2014). Spinal Cord Injury (SCI) Facts and Figures at a Glance. Spinal Cord Injury Model System: https://www.nscisc.uab.edu/PublicDocuments/fact_figures_docs/ Facts\%202014.pdf.

37. Hachen, H.J. (1977). Idealized care of the acutely injured spinal cord in Switzerland. J Trauma. 17, 931-936.

38. Gschaedler, R., Dollfus, P., Mole, J.P., Mole, L., and Loeb, J.P. (1979). Reflections on the intensive care of acute cervical spinal cord injuries in a general traumatology centre. Paraplegia 17, 58-61.

39. Kruger, E.A., Pires, M., Ngann, Y., Sterling, M., and Rubayi, S. (2013). Comprehensive management of pressure ulcers in spinal cord injury: current concepts and future trends. J. Spinal Cord Med. 36, 572-585.

40. Barat, M., Dehail, P., and de Seze, M. (2006). Fatigue after spinal cord injury. Ann. Readapt. Med. Phys. 49, 277-282, 365-369.

41. Aarabi, B., Harrop, J.S., Tator, C.H., Alexander, M., Dettori, J.R., Grossman, R.G., Fehlings, M.G., Mirvis, S.E., Shanmuganathan, K., Zacherl, K.M., Burau, K.D., Frankowski, R.F., Toups, E., Shaffrey, C.I., Guest, J.D., Harkema, S.J., Habashi, N.M., Andrews, P., Johnson, M.M., and Rosner, M.K. (2012). Predictors of pulmonary complications in blunt traumatic spinal cord injury. J. Neurosurg. Spine 17, Suppl 1, 38-45.

42. Sperry, J.L., Minei, J.P., Frankel, H.L., West, M.A., Harbrecht, B.G., Moore, E.E., Maier, R.V., and Nirula, R. (2008). Early use of vasopressors after injury: caution before constriction. J. Trauma 64 9-14.

Address correspondence to: Geoffrey Manley, MD, PhD Department of Neurological Surgery University of California, San Francisco 1001 Potrero Avenue, Building 1, Room 101 San Francisco, CA 94110

E-mail: manleyg@neurosurg.ucsf.edu 